\title{
Taller de sueño infantil. Herramientas para su interpretación por el pediatra de Atención Primaria
}

\author{
G. Pin Arboledas ${ }^{a}$, R. Ugarte Libanob \\ aPediatra. Unidad Valenciana del Sueño. Hospital Quirón. Valencia. España.

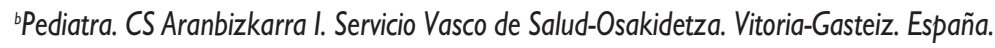

Rev Pediatr Aten Primaria. 2009; I I (Supl I 7):s399-s404

Gonzalo Pin Arboledas, gpa@comv.es

\section{Resumen}

Los trastornos del sueño son muy frecuentes en la edad pediátrica y pueden ocasionar alteraciones importantes a los niños y a sus familias. El pediatra de Atención Primaria es el profesional más adecuado para detectar, orientar, en muchos casos tratar, y siempre evaluar las consecuencias de estos trastornos. Para ello es necesario conocer las herramientas básicas de que se deben disponer en la consulta para la detección y evaluación de estos trastornos, habitualmente infradiagnosticados. Es primordial conocer los cuestionarios de cribado de patología relacionada con el sueño para administrarlos en el programa de salud infantil y los cuestionarios específicos para evaluar las alteraciones del sueño pediátrico más frecuentes y más relevantes en lo referente a morbilidad, como son los trastornos respiratorios durante el sueño. Otra herramienta de fácil uso y de enorme información son las agendas de sueño.

Con este taller se pretende concienciar de la importancia de estructurar en los programas de salud infantil el conocimiento sobre las características del sueño y sus posibles trastornos, así como adquirir habilidades prácticas con los cuestionarios de sueño más utilizados y en el manejo de las agendas de sueño.

Palabras clave: Trastornos del sueño, Cuestionarios de sueño, Agenda de sueño, Trastornos respiratorios durante el sueño, Programa de salud infantil.

Abstract

Sleep disorders in children and adolescents are very common and are often a cause of distress and concern for both the child and the family. The Primary Care Paediatrician is the most competent professional to detect, give advice, in many instances to treat, and always to evaluate the consequences of these disorders. For this purpose it is necessary to know the basic tools to detect and treat these problems, often under diagnosed in paediatric practice. It is important to be aware of screening tests and questionnaires to detect and evaluate the most frequent sleep disorders, and particularly sleep disordered breathing. A sleep diary is a simple tool for providing useful information about sleep problems in toddlers, children and adolescents.

Los autores declaran no presentar posibles conflictos de intereses en relación con la preparación y publicación de este artículo. 
The aim of this workshop is to become aware of the importance of sleep disorders in childhood and at the same time, introduce and manage these basic tools (screening tests, questionnaires and sleep diary) in the paediatric clinic, especially in the Paediatric Health Program.

Key words: Sleep disorders, Sleep questionnaires, Sleep diary, Sleep disordered breathing, Paediatric health program.

\section{Conceptos teóricos básicos de autorregistros y cuestionarios}

El concepto de calidad de sueño es un constructo en el que el uso de autorregistros, cuestionarios y observación de la conducta espontánea tienen un gran papel en su evaluación, diagnóstico y tratamiento.

\section{Autorregistros}

Un autoinforme supone un mensaje que un sujeto emite sobre cualquier tipo de manifestación propia. En el caso de los niños lo suelen realizar padres o tutores. El autorregistro exige atender deliberadamente a la conducta que se valora y registrarla.

A la hora de utilizar autorregistros es necesario reconocer que estos pueden inducir efectos reactivos sobre el paciente y su entorno (la llamada reactividad de los autorregistros). Los efectos positivos de esta reactividad, en ocasiones, son muy marcados y algunos factores la aumentan:

- La frecuencia de la autoobservación.
- Cuando la conducta a registrar es fácil y hay una alta probabilidad de éxito, registrar aspectos negativos de la conducta o el ambiente aumenta los efectos reactivos. Por el contrario, cuando la tarea o conducta es difícil, el registro de aspectos positivos puede facilitar los efectos reactivos.

- La motivación para el cambio.

El uso de los autorregistros debe quedar justificado por una sospecha diagnóstica clara que nos oriente hacia qué categorías incluir, así como el tiempo y momento del registro. Todo ello teniendo en cuenta que los autorregistros son tediosos y exigen dedicar un tiempo, por lo que es preferible, en ocasiones, hacerlos más sencillos aunque sea a costa de perder información.

\section{Cuestionarios}

Los cuestionarios nos van a permitir obtener datos de las características del funcionamiento de los sujetos.

Los cuestionarios son básicamente una forma de sistematizar la búsqueda de da- 
tos $y$, como tal, son instrumentos de evaluación adecuados para valorar los aspectos subjetivos-cognitivos de la materia objeto del análisis (el sueño) y presentan determinadas ventajas: sistematización, facilidad de aplicación, economía y posibilidad de referencia interindividual.

El uso de los cuestionarios exige preguntarse previamente por el objetivo que se pretende lograr con la utilización de un determinado cuestionario. El objetivo, en ocasiones, no solo es la identificación de una determinada conducta, sino también la motivación del paciente y el manejo o conocimiento de sus expectativas. Así, los objetivos de un cuestionario podrían quedar perfilados como:

- Obtención de información dirigida a la confección de un programa de tratamiento. Se busca fundamentalmente lo cualitativo, lo descriptivo y lo singular.

- Clasificación diagnóstica.

- Variables predictivas de la eficacia del tratamiento.

- Motivación terapéutica.

Al utilizar los cuestionarios debemos tener presentes algunas consideraciones:

1. El cuestionario produce un efecto reactivo e influencia el comportamiento del paciente. Lo debemos tener presente a efectos de la evaluación y evitar su uso abusivo, que podría potenciar un posible efecto reactivo adverso.

2. En ocasiones no es conveniente utilizar las puntuaciones obtenidas como un dato inapelable en la intervención terapéutica.

3. Deben utilizarse siempre en el marco de la evaluación general del paciente y en conjunción con otras pruebas complementarias o clínicas.

4. Deben elegirse aquellos cuestionarios que tengan mayores garantías científicas en términos de fiabilidad y validez.

\section{Observación de la conducta}

Es básica en la evaluación del niño con problemas de o con el sueño. Su objetivo es obtener información acerca del comportamiento del niño y su entorno. Los elementos objetos de la información se pueden clasificar como:

a) Conductas: del niño y los padres o tutores durante la entrevista de manera separada.

b) Interacciones de los padres o tutores y su modelo educativo (permisivo...).

c) Resultado de estas interacciones en la conducta del niño, los padres o tutores y su interrelación.

\section{Agendas de sueño}

Son sencillas, fáciles de cumplimentar por las familias y proporcionan una infor- 
mación global del sueño en los niños. Es necesario registrar un mínimo de dos semanas para tener una información adecuada.

Las agendas de sueño son, en alguna medida, comparables a la actigrafía, aunque presentan limitaciones a la hora de registrar los despertares nocturnos en niños pequeños ${ }^{1}$. El uso combinado de actigrafía y agendas de sueño es de utilidad en la valoración de la cantidad y eficiencia de sueño, sobre todo en casos de somnolencia ${ }^{2}$ y también en el trastorno por déficit de atención e hiperactividad ${ }^{3}$.

\section{Cuestionarios de sueño}

Los cuestionarios de sueño pueden ser de utilidad tanto en niños sanos como en aquellos con trastornos del comportamiento ${ }^{4}$ y pueden aplicarse desde el período neonatal.

Hay cuestionarios de cribado rápido, sencillos, cuyo uso podría sistematizarse en los programas de salud infantil para detectar trastornos del sueño, y otros más amplios indicados para orientar en problemas de sueño. Desde las primeras consultas en Atención Primaria puede emplearse en recién nacidos y lactantes el Brief Screening Questionnaire for Infant Sleep Problems, más conocido por su acrónimo BISQ ${ }^{5}$. Su utilidad va dirigida a detectar factores de riesgo de muer- te súbita del lactante, rutinas para dormir y detección de problemas de sueño en lactantes por parte de los padres. Sadeh, su autor, encontró una correlación significativa entre la actigrafía y los datos obtenidos con este test en lactantes de 5 a 29 meses de edad en el número de despertares nocturnos y la duración del sueño nocturno, por lo que es una buena herramienta de cribado. Fue desarrollado con base en las variables significativas encontradas en una revisión de la literatura; estas variables fueron: duración del sueño nocturno (entre las 19 a las 7 horas [h]), duración del sueño diurno (entre las 7 y las $19 \mathrm{~h}$ ), número de despertares nocturnos, duración de los despertares nocturnos (entre las 22 y las $6 \mathrm{~h}$ ), hora de dormir, duración de la latencia del sueño, método para quedarse dormido, lugar en que duerme, posición corporal preferida, edad del niño, sexo, lugar en la fratría y persona que contesta el cuestionario. Es importante conocer estos datos, ya que en nuestro país los horarios son diferentes, más tardíos, y no ha sido validado en nuestro ámbito. Se requiere entre 5-10 minutos para cumplimentar el cuestionario.

A partir de los 2 años el cuestionario $\mathrm{BEARS}^{6}$ es una buena herramienta de cribado. Este cuestionario valora cinco aspectos del sueño: problemas para acos- 
tarse, excesiva somnolencia diurna, despertares nocturnos, regularidad y duración del sueño y ronquido. Este cuestionario se administra en tres tramos de edad: 2 a 5 años, 6 a 12 años y 13 a 18 años, con preguntas dirigidas a padres $y$ a los niños mayores.

Hay otros cuestionarios generales, más amplios que los anteriores, como es el Cuestionario de Hábitos de Sueño de Owens (Children's Sleep Habits Questionnaire o $\mathrm{CSHQ}^{7}$ ).

La escala de Trastornos del Sueño para Niños de Bruni (Sleep Disturbance Scale for Children, SDSC) ${ }^{8}$ consta de 27 ítems valorados según una escala tipo Likert y está diseñada para detectar trastornos del sueño. Evalúa los últimos 6 meses. Su consistencia interna es mayor en los controles $(0,79)$, se mantiene con un nivel satisfactorio en los niños con trastornos del sueño $(0,71)$ y la fiabilidad es satisfactoria para el total $(r=0,71)$ y para cada reactivo de forma individual.

El Cuestionario de Sueño Pediátrico de Chervin (Pediatric Sleep Questionnaire o PSQ $)^{9}$ tiene dos versiones. Una reducida, con 22 preguntas, orientada a los trastornos respiratorios durante el sueño y que es el referente en la sospecha de síndrome de apnea hipopnea de sueño (SAHS), según se acordó en el consenso nacional del SAHS ${ }^{10}$. Sus medidas de validez, fiabilidad y sensibilidad son superiores a 0,80 . Una de sus características es que compara los síntomas de inatención e hiperactividad y además los correlacionó con los hallazgos de la polisomnografía. Este cuestionario ha sido traducido y validado en lengua española"1.

Hay otros cuestionarios dirigidos a procesos concretos como los trastornos respiratorios durante el sueño como el ya comentado PSQ o el videoscore de Si$\operatorname{van}^{12}$ y el de calidad de vida en el SAHS ${ }^{13}$, el síndrome de piernas inquietas, excesiva somnolencia diurna, matutinidad y vespertinidad, etc.

En adolescentes es muy útil, no está validado en castellano, el School Habits Survey original de Carskadon ${ }^{14,15}$. Evalúa durante las dos últimas semanas de su aplicación el sueño, la somnolencia, el uso de sustancias, la ansiedad, el ánimo depresivo, el uso de fármacos para dormir, la asistencia escolar y las preferencias circadianas.

La mayoría de estos cuestionarios y un modelo de agenda de sueño están accesibles desde la página web del Grupo de Sueño de la AEPap ${ }^{16}$. 


\section{Bibliografía}

1. Werner $H$, Molinari L, Guyer C, Jenni OG. Agreement rates between actigraphy, diary, and questionnaire for children's sleep patterns. Arch Pediatr Adolesc Med. 2008;162(4):350-8.

2. Kushida CA, Chang A, Gadkary C, Guilleminault Ch, Carrillo O, Dement WC. Comparison of actigraphic, polysomnographic, and subjective assessment of sleep parameters in sleep-disordered patients.Sleep Medicine. 2001;2(5):389-96.

3. Owens JA, Sangal RB, Sutton VK, Bakken R, Allen AJ, Kelsey D. Subjective and objective measures of sleep in children with attention-deficit/hyperactivity disorder Sleep Medicine. 2009;1(4): 446-56.

4. Goodlin-Jones BL, Sitnick SL, Tang K, Liu J, Anders TF. The children's sleep habits questionnaire in toddlers and preschool children. J Dev Behav Pediatr. 2008;29(2):82-8.

5. Sadeh A. A brief screening questionnaire for infant sleep problems: validation and findings for an internet sample. Pediatrics. 2004;113(6): e570-7.

6. Owens JA, Dalzell V. Use of the 'BEARS' sleep screening tool in a pediatric residents' continuity clinic: a pilot study. Sleep Medicine. 2005; 6(1):63-9.

7. Owens JA, Spirito A, McGuinn M. The Children's Sleep Habits Questionnaire (CSHQ): psychometric properties of a survey instrument for school-aged children. Sleep. 2000;23(8):1043-51.

8. Bruni O, Octaviano S, Guidetti V, Romoli $M$, Innocenzi M, Cortesi F, et al. The Disturbance Sca- le for Children (SDSC). Construction and validation of an instrument to evaluate sleep disturbances in childhood and adolescence. J Sleep Res. 1996;5: 251-61.

9. Chervin RD, Hedger K, Dillon JE, Pituch KJ. Pediatric sleep questionnaire (PSQ): validity and reliability of scales for sleep-disordered breathing, snoring, sleepiness, and behavioral problems. Sleep Med. 2000;1(1):21-32.

10. Consenso Nacional sobre el síndrome de apneas-hipopneas del sueño [consultado el 12/09/ 2009]. Disponible en www.aepap.org/pdf/sahs completo.pdf

11. Tomás Vila $M$, Miralles Torres A, Beseler Soto B. Versión española del Pediatric Sleep Questionnaire. Un instrumento útil en la investigación de los trastornos del sueño en la infancia. Análisis de su fiabilidad. An Pediatr (Barc). 2007;66:121-8.

12. Sivan $Y$, Kornecki A, Schonfeld T. Screening obstructive sleep apnoea syndrome by home videotape recording in children. Eur Respir J. 1996;9: 2127-31.

13. De Serres LM, Derkay C, Astley S, Deyo RA, Rosenfeld RM, Gates GA. Arch Otolaryngol Head Neck Surg. 2000;126:1423-9.

14. Carskadon MA, Seifer R, Davis $S$, Acebo $C$. Sleepiness in college-bound high school seniors. Sleep Res. 1991;20:175.

15. Carskadon MA, Seifer R, Davis $S$, Acebo $C$. Reliability of six scales in a sleep questionnaire for adolescents. Sleep Res. 1991;20:421.

16. Grupo de Sueño de la AEpap [consultado el 12/09/2009]. Disponible en www.aepap.org/gt siaepap/?page_id=9 